\title{
Dreizähniges Knabenkraut (Neotinea tridentata), Orchidee des Jahres 2019
}

\author{
Mathias Lohr \& Bernd Margenburg
}

\begin{abstract}
The three-toothed orchid (Neotinea tridentata) is orchid of the year 2019. The species is widespread in the Mediterranean, occurs also in Germany and is also known from Hesse. There are natural hybrids between Neotinea tridentata and the burnt orchid (Neotinea ustulata), both recently separated from the genus Orchis and placed in the genus Neotinea. Biology and ecology of this orchid are presented.
\end{abstract}

\section{Zusammenfassung}

Das Dreizähnige Knabenkraut (Neotinea tridentata) ist Orchidee des Jahres 2019. Die Art ist im Mittelmeerraum weit verbreitet, kommt stellenweise, so auch in Hessen, aber auch in Deutschland vor. Hier ist es eine gefährdete Art. Es gibt natürliche Hybriden zwischen dem Dreizähnigen Knabenkraut und dem Brand-Knabenkraut (Neotinea ustulata), die beide erst seit kurzem aus der Gattung Orchis ausgegliedert und zur Gattung Neotinea gestellt wurden. Biologie und Ökologie dieser Orchidee werden vorgestellt.

\section{Einleitung}

Um auf die Schönheit und die Schutzwürdigkeit sowie auf die Veränderungen ihrer Lebensräume und bei vielen Arten auf Bestandsrückgänge aufmerksam zu machen, wird jährlich von den deutschen „Arbeitskreisen Heimische Orchideen“" (AHO Deutschland) eine Orchidee des Jahres gewählt. Im Jahr 2019 fiel die Wahl auf das Drei- zähnige Knabenkraut (Neotinea tridentata, oft noch besser bekannt unter dem älteren Namen Orchis tridentata).

\section{Name}

Neotinea tridentata wurde im Jahr 1772 von dem österreichischen Arzt und Naturforscher Giovanni Antonio Scopoli in der Flora Carniolica zur

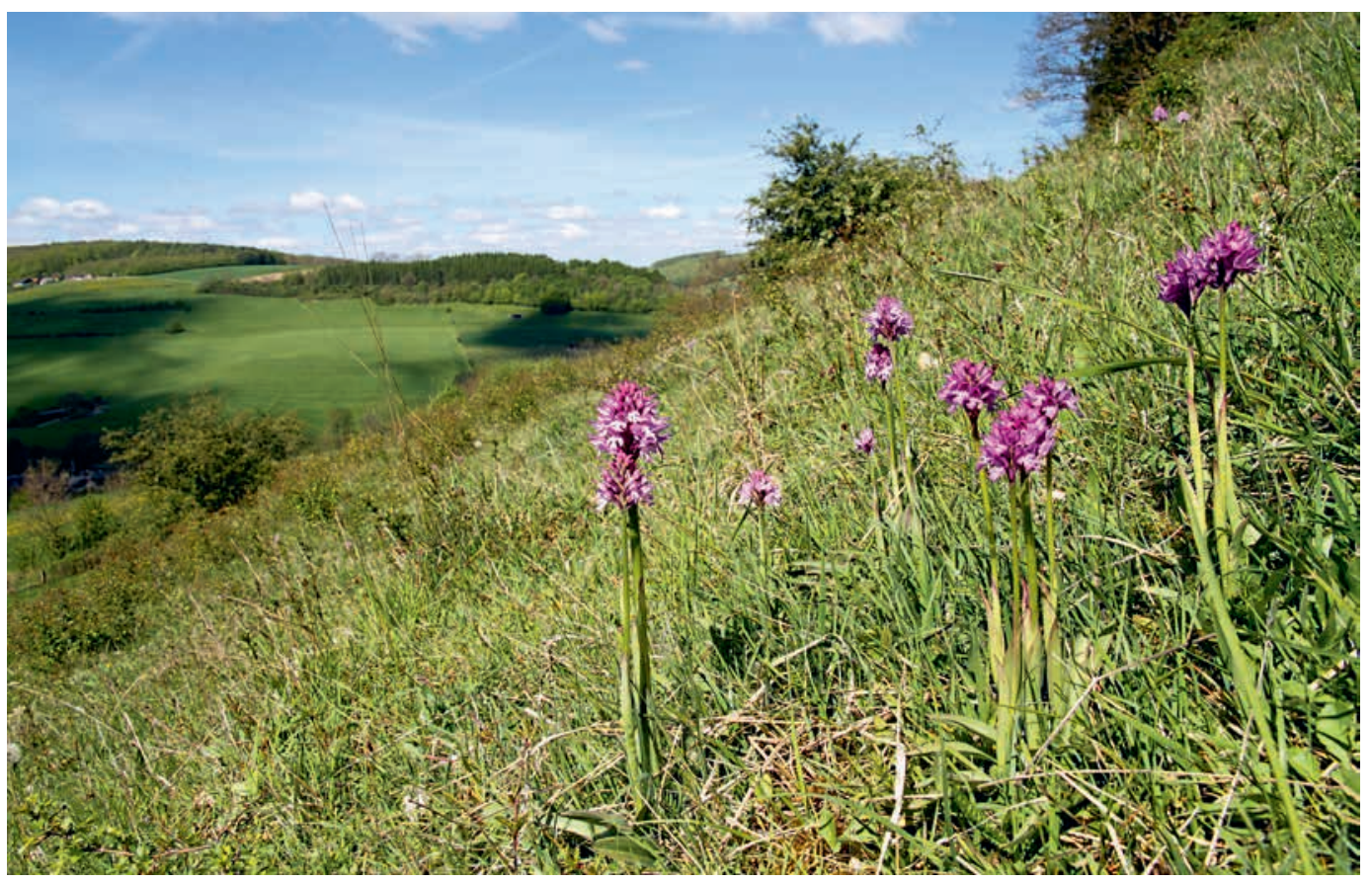

Abb. 1: Neotinea tridentata auf einem Kalkmagerrasen im Kreis Höxter, 9.5. 2017 (Foto: M. LoHR) 
Gattung Orchis gestellt und als Orchis tridentata beschrieben. Der Gattungsname Orchis leitet sich vom griechischen Wort für Hoden ab und bezieht sich auf die Form der beiden Wurzelknollen. Der lateinische Artbeiname tridentata bedeutet dreizähnig (lat. tri = drei; dens = Zahn). Nach einigen Autoren bezieht sich diese Bezeichnung auf die Anzahl der spitz zulaufenden, nach außen gebogenen Zipfel der Sepalen (äußere Blütenblätter), die somit einen dreispitzigen Helm bilden. Andere halten die dreilappige Lippe für namengebend (z. B. AHO 2005). Der aktuelle Gattungsname Neotinea wurde zu Ehren des italienischen Botanikers Vincenzo Tineo (1791-1856) vergeben, der Professor in Palermo war.

Genetische Untersuchungen von BATEMAN et al. (1997) führten dazu, dass das Dreizähnige Knabenkraut mit nahe verwandten Arten in die Gattung Neotinea überführt wurde. Tyteca \& KLEIN (2008) halten, wie auch andere Autoren, die Einordnung zu Neotinea für falsch und beschrieben die neue Gattung Odontorchis, in die sie die Art als Odontorchis tridentata stellten. $\mathrm{Ob}$ die Zuordnung der Art zu der ursprünglich mono- typischen Gattung Neotinea, die u. a. um die ehemaligen Orchis-Arten O. tridentata, O. ustulata und $O$. lactea erweitert wurde, oder zu einer neuen Gattung Odontorchis die tatsächlichen taxonomischen Verhältnisse besser widergibt, werden weitere Untersuchungen zeigen müssen.

\section{Verbreitung und Lebensräume}

Neotinea tridentata ist in verschiedenen Regionen Deutschlands unterschiedlich häufig verbreitet. In Nordrhein-Westfalen kommt sie fast ausschließlich im südlichen Teil des Weserberglandes vor. Die ostwestfälischen Vorkommen von Neotinea tridentata gehören zu einem vom weiter südlich liegenden Hauptverbreitungsgebiet isolierten Teilareal, das außerdem Teile Thüringens, Sachsen-Anhalts, Hessens und Südniedersachsens umfasst. Ein weiteres, deutlich kleineres Teilareal liegt im östlichen Brandenburg an der Oder. Neotinea tridentata gilt als Art mit submediterranem Verbreitungsschwerpunkt. Das Hauptareal erstreckt sich vom Kaukasus über die Schwarzmeerküsten, den Balkan, Italien und Südfrankreich bis an den Südrand der Alpen und der Karpaten.

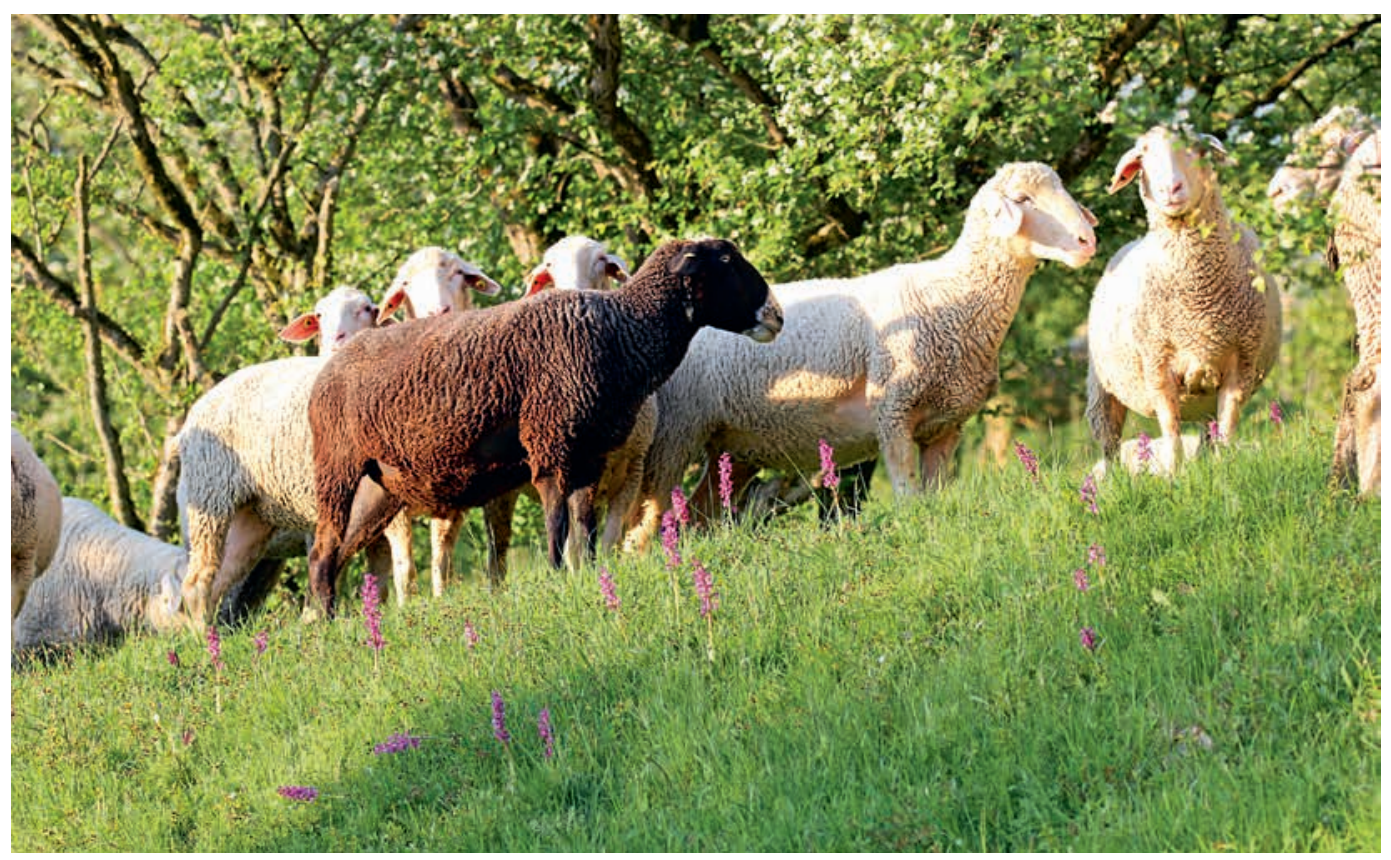

Abb. 2: Schafbeweidung auch zur Blütezeit trägt zum Erhalt der Lebensräume von Orchideen der Kalkmagerrasen bei, hier von Orchis mascula, Kreis Höxter, 1.5. 2018. (Foto: M. LoHR) 


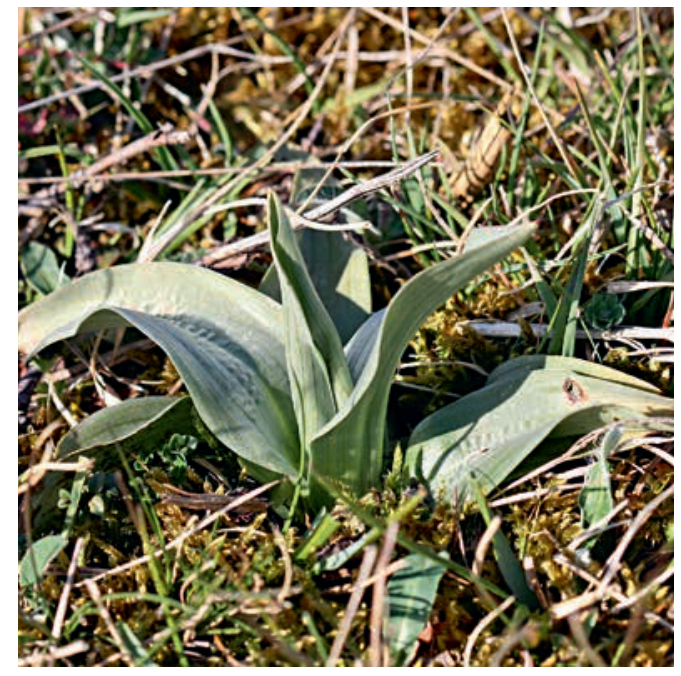

Abb. 3: Austrieb von Neotinea tridentata im Kreis Höxter, 22.4. 2007. (Foto: M. LoHr)

Die Lebensräume von Neotinea tridentata sind bevorzugt offene Kalkmagerrasen, die extensiv von Schafen oder Rindern beweidet werden. Die höchsten Dichten erreicht die Art in leicht versaumten Beständen. Nach Aufgabe der Beweidung hält sie sich oft noch einige Jahre, verschwindet dann aber mit zunehmender Verfilzung besonders durch die Fieder-Zwenke (Brachypodium pinnatum). Nach Haeupler \& Muer (2000) kommt die Art in Pflanzengesellschaften der Trespen-Halbtrocken-

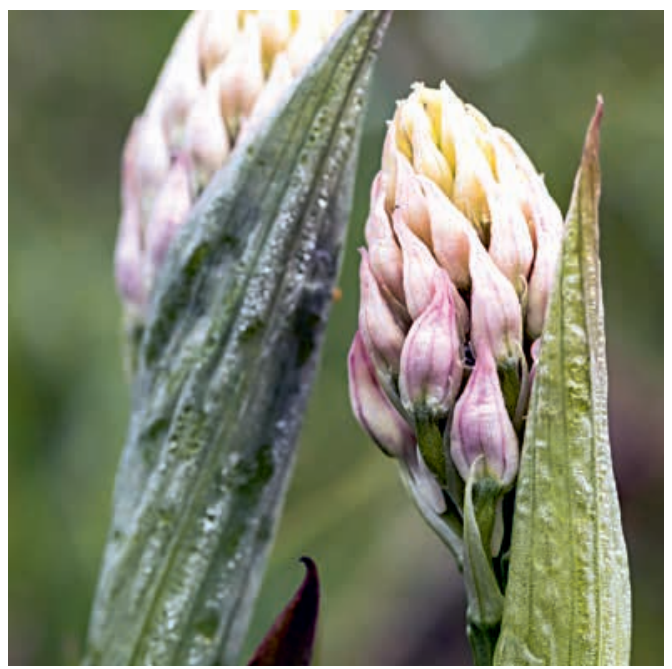

Abb. 4: Knospen von Neotinea tridentata im Kreis Höxter, 4.5. 2015. (Foto: W. KuHN). rasen (Mesobromion erecti) und wärmebedürftigen Blutstorchschnabel-Säumen (Geranion sanguinei) vor.

Neotinea tridentata gilt als Kennart der beweideten Enzian-Schillergrasrasen (Gentiano-Koelerietum pyramidatae, OBERdorfer 2001). Im Weserbergland besteht der geologische Untergrund der Vorkommen zumeist aus Muschelkalk, in Hessen und Thüringen kommt die Art auch auf Zechstein vor. Die flachgründigen Böden, in der Regel Rendzinen, sind sehr stickstoffarm und kalkreich. Neotinea tridentata ist ein Zeiger deutlich nährstoffarmer Standorte. Ellenberg (1974) weist ihr entsprechend die Stickstoffzahl 2 zu, als Basen- und Kalkzeiger hat sie die Reaktionszahl 9 und als Trockenheitszeiger die Feuchtezahl 3. Als Volllichtpflanze weist sie eine Lichtzahl von 9 und als Wärmezeiger die Temperaturzahl 7 auf. Sie bevorzugt mäßig geneigte, mäßig bis voll besonnte Südhänge mit einer geringmächtigen Streufilzauflage. Sundermann (1980) nennt für die Bodensubstrate einen $\mathrm{pH}$-Wert von 6,3 bis 7,8 .

\section{Morphologie und Biologie}

Die kleinen bis mittelgroßen, aber kräftigen Pflanzen erreichen eine Wuchshöhe von 15 bis 30 , ausnahmsweise bis $45 \mathrm{~cm}$. Die silbrig-blau-

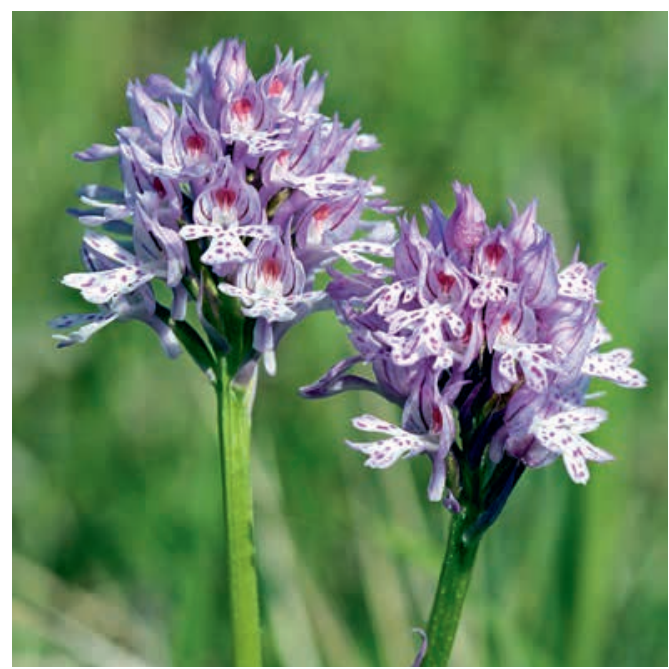

Abb. 5: Blütenstände von Neotinea tridentata im Kreis Höxter, 9.5. 2018 (Foto: M. Lohr). 
grünen lanzettlichen Laubblätter treiben bereits im Herbst aus und die Art bildet, wie viele andere submediterran verbreitete Orchideenarten, eine Winterblattrosette. Diese befähigt sie zur Assimilation auch im Herbst und Winter während günstiger Witterungsphasen (AHO Thüringen 2014). Drei bis fünf Blätter bilden eine grundständige Rosette, aus der im Frühjahr der Blütentrieb herauswächst. Der blaugrüne Stängel ist von mehreren Stängelblättern weitgehend umschlossen. Der Blütenstand von Neotinea tridentata ist dichtblütig, meist eiförmig bis halbkugelig und bei kräftigen Pflanzen walzenförmig verlängert. Die schmalen Tragblätter sind etwa so lang wie der Fruchtknoten. Die rosa bis rosaroten Sepalen und Petalen sind rot geädert und zu einem dreispitzigen Helm zusammengezogen (Name!). Selten kommen auch reinweiße Exemplare vor. Die tief dreilappige Lippe ist hellrosa bis weiß und gepunktet. Der zweiteilige Mittellappen hat in der Bucht ein Zähnchen. Die Ränder von Seiten- und Mittellappen sind häufig leicht aufwärts gewölbt. Der zylindrische Sporn, mit schmalem, wulstigem Sporneingang, ist leicht abwärts gebogen und etwa halb so lang wie der Fruchtknoten. Er enthält keinen Nektar. Die langgestielten Pollinarien sind gelblichbraun und haben getrennte Klebscheiben.

Neotinea tridentata gehört zu den Nektartäuschblumen, die den Blütenbesuchern ein Nektarangebot nur vortäuschen. Zur Bestäubung werden vor allem Bienen angelockt. Neben der Honigbiene (Apis mellifera) wurden zahlreiche Wildbienenarten als Bestäuber festgestellt, darunter Andrena cineraria, Bombus hortorum, $B$. terrestris, Halictus patellatus, Lasioglossum spec., Nomada fucata, Osmia aurulenta und O. bicolor (Überblick in Claessens \& Kleynen 2016). Die Beobachtung von Halictus patellatus als Bestäuber stammt aus Süditalien, in Deutschland wurde das Insekt bislang nicht nachgewiesen (Cozzolino et al. 2005, Westrich 2018). Außerdem werden die Blüten auch regelmäßig von Käfern (Coleoptera) und Zweiflüglern (Diptera) besucht. In Nordrhein-Westfalen wurden aus diesen Gruppen z. B. der Goldglänzende Rosenkäfer (Cetonia aurata) und die Schwarze Tanzfliege (Empis ciliata) als Bestäuber beobachtet (LoHr 2018).

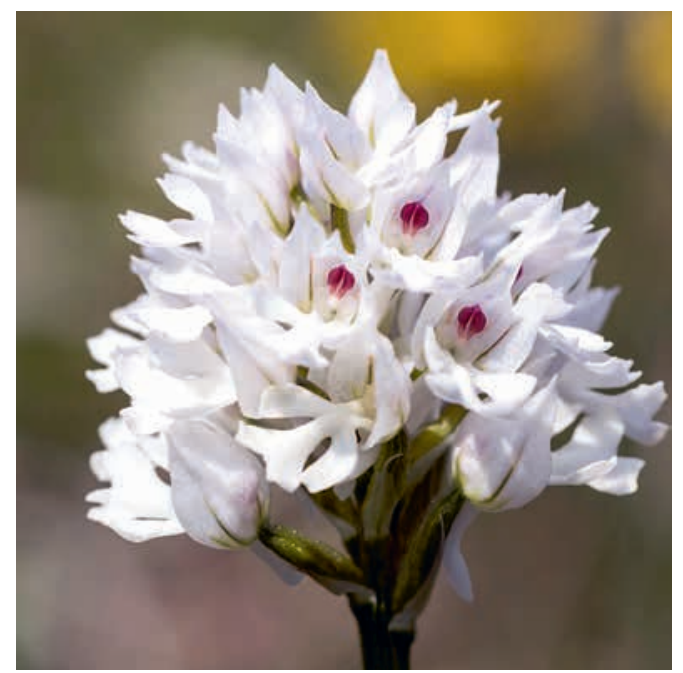

Abb. 6: Neotinea tridentata mit weißen Blüten in Thüringen, 12.5. 2016. (Foto: W. KuHN)

Insekten werden von Neotinea tridentata durch einen honig- und vanilleartigen Duft angelockt. Die meist unregelmäßig auf der Lippe angeordneten Punkte haben die Funktion von Saftmalen, die Blütenbesucher zum Sporneingang leiten, um mit vermeintlich dort vorhandenem Nektar belohnt zu werden. Dabei entnehmen sie Pollinien oder streifen sie ab (Claessens \& KLeynen 2011).

Neben Bestäubern und anderen Blütenbesuchern finden sich auf den Blütenständen von Neotinea tridentata regelmäßig auch räuberisch lebende Wirbellose. Am häufigsten ist wohl die Veränderliche Krabbenspinne (Misumena vatia) vertreten. Der Anteil fruchtender Kapseln kann stark schwanken und dürfte vor allem von den jeweiligen Witterungsbedingungen abhängig sein. Nach Claessens \& Kleynen (2011) liegt er zwischen 10 und $70 \%$. Die Früchte sind Kapseln, die hygroskopisch verschließbare Längsspalten aufweisen. Die winzig kleinen Samen, die nur aus dem Embryo und wenigen Zellen bestehen, sind mit Luftsäcken ausgestattet. Bei trockenwarmer Witterung können sie vom Wind über weite Strecken transportiert werden. Neotinea tridentata kann sich bei Verbiss auch vegetativ vermehren, indem sie basal Brutknospen bildet und im Folgejahr mit mehreren, nahe beieinander wachsenden Blütenständen austreibt (SIEBERT 1998). 


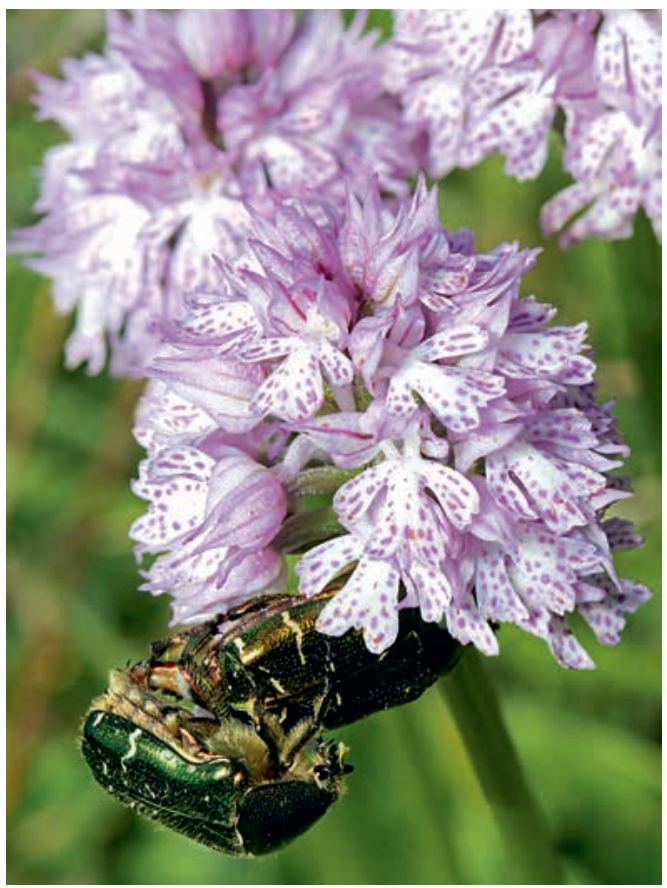

Abb. 7: Käfer (hier der Goldglänzende Rosenkäfer Cetonia aurata) treten bei Blütenpflanzen meist als Pollenfresser auf, die dadurch auch zur Bestäubung der Pflanzen beitragen, da sie in der Regel nicht alle Pollenkörner verzehren; dieses Pärchen hat sich auf dem Blütenstand zur Kopulation abgesetzt und vermutlich nur zufällig Pollinarien entnommen. Aufnahme aus dem Kreis Höxter, 3.5. 2014. (Foto: M. LoHr).

\section{Gefährdung}

Das Dreizähnige Knabenkraut ist eine geschützte Art. In Niedersachsen, Thüringen und Sachsen-Anhalt gilt es als stark gefährdet und in Brandenburg als vom Aussterben bedroht. In Hessen wird es als Art der Vorwarnliste geführt, d. h. als Art, die merklich zurückgegangen, aber aktuell noch nicht gefährdet ist. Bei Fortbestehen der bestandsreduzierenden Einwirkungen ist in naher Zukunft dort aber auch eine Einstufung in die Kategorie gefährdet wahrscheinlich. In der im Jahr 2018 vom Bundesamt für Naturschutz neu veröffentlichten Roten Liste Deutschlands wird Neotinea tridentata als gefährdet (= RL 3) geführt (Bundesamt für Naturschutz 2018).

Häufigste Gefährdungsursache ist die Verfilzung und Verbuschung der Kalkmagerrasen infolge der Aufgabe von Beweidung. Auch durch Eutrophierung der Lebensräume durch Stickstoffeintrag von benachbarten Ackerflächen oder aus der Luft dürften Vorkommen verloren gegangen sein.

\section{Schutz und Entwicklungsmaßnahmen}

Schutzmaßnahmen für die Art umfassen vor allem den Erhalt und die Optimierung ihrer Lebensräume. Dazu ist besonders die Aufrechterhaltung oder Wiederaufnahme der Beweidung durch Schafe und Ziegen oder, auf weniger steilen Hängen, auch extensiv durch Rinder zu gewährleisten. Auf länger aus der Nutzung genommenen Flächen sind ggf. zuvor Entbuschungsmaßnahmen durchzuführen. Optimal für die Art ist die extensive Hütehaltung in Anlehnung an die historische Nutzungsform der Wanderschäferei mit geeigneten Schafrassen. Auch eine intensivere Schafbeweidung scheint der Art nicht zu schaden, wenngleich sich eine intensive Koppelhaltung nachteilig auf die Bestände der Art auswirkt (Lohr \& Grawe 2005).

Die jahrweise Beweidung zur Blütezeit führt langfristig nicht zum Rückgang der Art, da sie sich auch vegetativ gut vermehren kann und im Folgejahr wieder zur Blüte kommt. Bestandszunahmen konnten beispielsweise durch Entbuschungsmaßnahmen und Wiederaufnahme der Schafbeweidung im Kreis Höxter erzielt werden (Lohr et al. 2018).

\section{Hybriden}

Neotinea tridentata hybridisiert in Deutschland nur mit Neotinea ustulata (= Orchis ustulata), dem Brand-Knabenkraut. Anders als in Thüringen kommt diese Hybride in Nordrhein-Westfalen nicht vor, da es in diesem Bundesland keine gemeinsamen Wuchsorte beider Arten gibt. Nach Angaben des AHO Thüringen werden Hybriden an fast allen gemeinsamen Fundorten beobachtet. Da gelegentlich Hybridschwärme auftreten, ist zu vermuten, dass auch die Nachkommen der Kreuzungen fruchtbar sind (KRETZsChmar et al. 2007). Die Bestimmung ist jedoch nicht einfach, insbesondere bei Berücksichtigung der Variationsbreite der typischen Merkmale beider Arten. Der Einfluss von Neotinea ustulata ist (relativ sicher) an der dunklen Helmfarbe zu erkennen. Die Blü- 


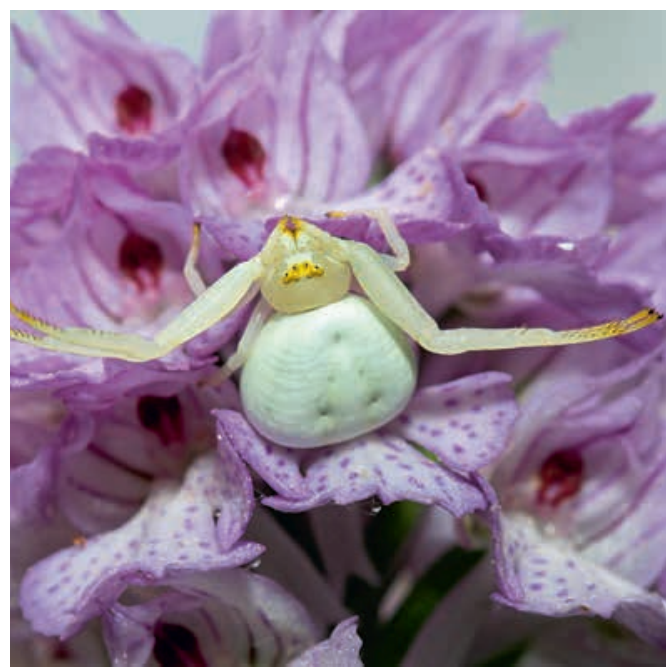

Abb. 8: Auf Blütenbesucher lauernde Veränderliche Krabbenspinne (Misumena vatia) auf einem Blütenstand von Neotinea tridentata im Kreis Höxter, 22.05.2016 (Foto: M. Lohr).

tengröße und die verlängerten Lappen der Lippe dagegen werden oftmals von Neotinea tridentata bestimmt.

Die Überführung von Orchis tridentata und Orchis ustulata in die Gattung Neotinea bedingte die Neukombination der Nothospecies: Neotinea $x$ dietrichiana (Bogenh.) H. Kretzschmar, Eccarius \& H. Dietr. Es handelt sich um eine Hybride zwischen Neotinea tridentata und Neotinea ustulata.

\section{Dank}

Wir danken Herrn Wilfried KuHn (Wuppertal) für die freundlicherweise zur Verfügung gestellten Fotos.

\section{Literatur}

Arbeitskreise Heimischer Orchideen DeutschLANDS (HrsG.) 2005: Die Orchideen Deutschlands. - Uhlstädt-Kirchhasel.

AHO NRW (Arbeitskreise Heimischer Orchideen Nordrhein-Westfalen) 2018: Die Orchideen NordrheinWestfalens. - Münster.

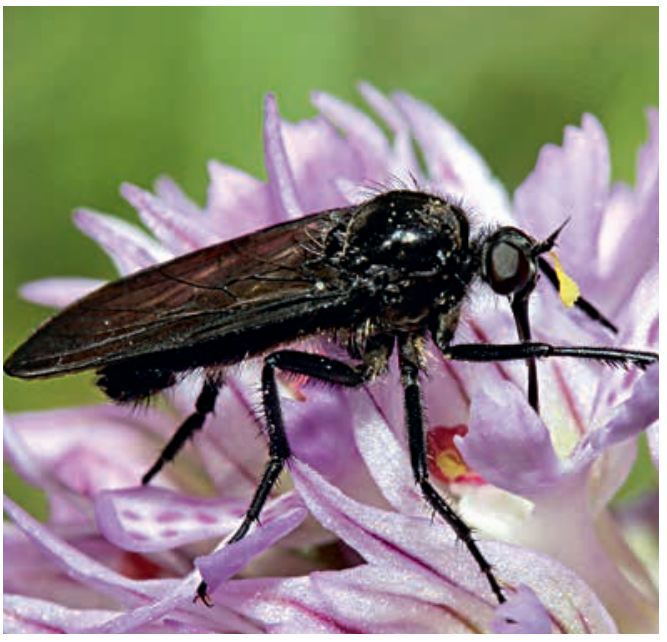

Abb. 9: Schwarze Tanzfliege (Empis ciliata), die mit ihrem langen Rüssel vergeblich in den Spornen von Neotinea tridentata-Blüten nach Nektar sucht, dabei jedoch ein Pollinarium entnommen hat. Beobachtung im Kreis Höxter, 3.5. 2014. (Foto. M. Lohr)

AHO Sachsen-Anhalt (Arbeitskreise Heimischer OrChideen Sachsen-Anhalt e. V.) (Hrsg.) 2011: Orchideen in Sachsen-Anhalt. Verbreitung, Ökologie, Variabilität, Gefährdung, Schutz. - Löbejün.

AHO Thüringen (Arbeitskreise Heimischer OrchiDeEn Thüringen) (Hrsg.) 2014: Thüringens Orchideen. Uhlstädt- Kirchhasel.

Bateman, R. M., Pridgeon, A. M. \& Chase M. W. 1997: Phylogenetics of subtribe Orchidinae (Orchidoideae, Orchidaceae) based on nuclear ITS sequences. 2. Infrageneric relationships and reclassification to achieve monophyly of Orchis sensu stricto. - Lindleyana 12: 113-141.

Bundesamt für naturschutz 2018: Rote Liste und Gesamtartenliste der Farn- und Blütenpflanzen (Trachaeophyta) Deutschlands. Naturschutz und Biologische Vielfalt 70. - Bonn Bad Godesberg.

Claessens, J. \& Kleynen, J. 2011: The flower of the European orchid - form and function. - Voerendaal.

Claessens, J. \& Kleynen, J. 2016: Orchidées d'Europe, fleur et pollinisation. - Mèze.

Cozzolino, S., Schiestl, F. P., Müller, A., Da Castro, O., Nardella A. M. \& Widmer A. 2005: Evidence for pollinator sharing in mediterranean orchids: absence of premating barriers? - Proc Royal Soc London, Ser. B, Biol. Sci 272: 1271-1278.

Ellenberg, H. 1979: Zeigerwerte der Gefäßpflanzen Mitteleuropas, 2. Aufl. - Scripta Geobot. 9.

Haupler, H. \& Muer, T. 2000: Bildatlas der Farn- und Blütenpflanzen Deutschlands. - Stuttgart. 


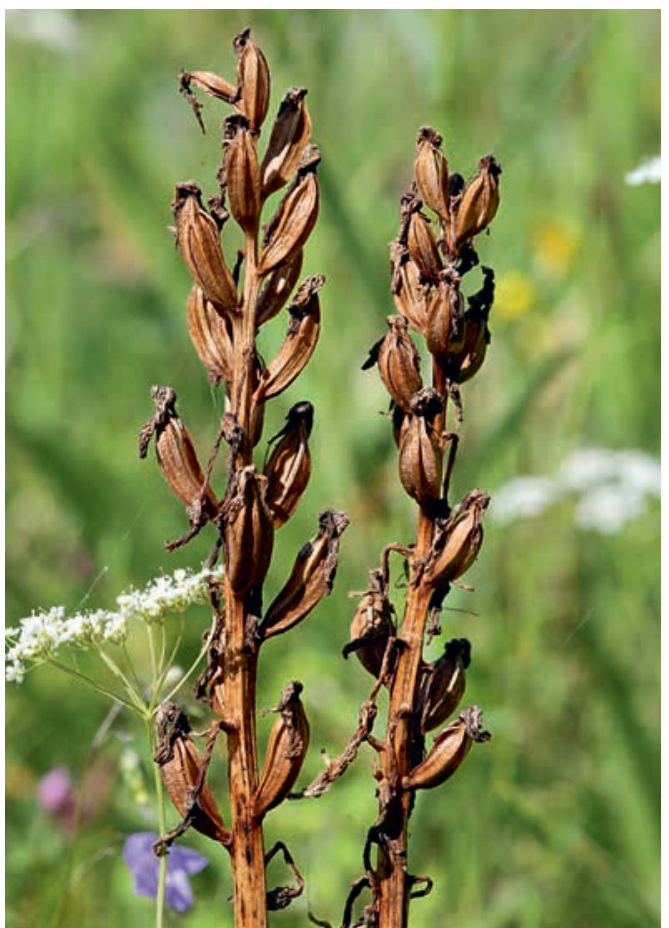

Abb. 10: Fruchtstände von Neotinea tridentata. Beobachtung im Kreis Höxer, 31.7. 2017. (Foto: M. Lohr)

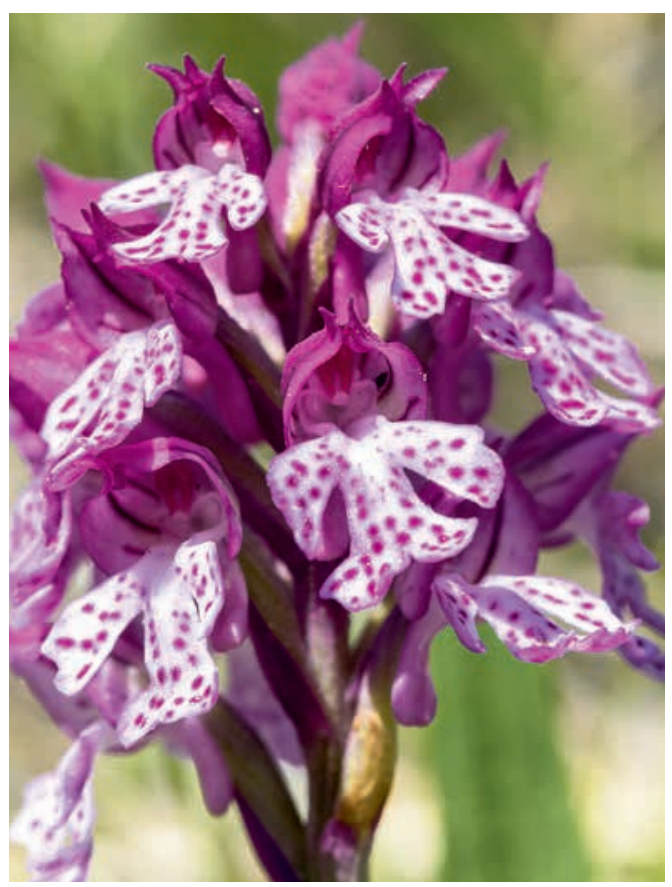

Abb. 11: Neotinea $\times$ dietrichiana (Orchis tridentata $\times$ ustulata) in Thüringen, 12.5. 2016. (Foto: W. KuHN).
Kretzschmar, H., Eccarius, W. \& Dietrich, H. 2007: Die Orchideengattungen Anacamptis, Orchis, Neotinea. - Bad Hersfeld.

Landesamt für Umwelt 2006: Rote Liste Gefäßpflanzen in Naturschutz und Landschaftspllege in Brandenburg 15: 70-80.

Lohr, M. \& Grawe, F. 2005: Das Dreizähnige Knabenkraut (Orchis tridentata) im Kreis Höxter. - Beiträge zur Naturkunde zwischen Egge und Weser 17: 123-128.

Lohr, M. 2018: Belohnen, Täuschen und Betrügen - von den Fortpflanzungsstrategien der Orchideen. - In: Kuhn, W., Küpper, D., Lohr, M., Loos, G. H., Luwe, M., Margenburg, B., Wenker, D., Westphal, G. \& Wolbeck, D.: Die Orchideen Nordrhein-Westfalens. - Münster.

Lohr, M., Beinlich, B., Grawe, F. \& Härtl, K.-H. 2018: Förderung von Orchideen im Kreis Höxter durch das Life+-Projekt „Vielfalt auf Kalk“. - In: Kiuhn, W., Küpper, D., Lohr M., Loos, G. H., Luwe, M., Margenburg, B., Wenker, D., Westphal, G. \& Wolbeck, D.: Die Orchideen Nordrhein-Westfalens. - Münster.

Oberdorfer, E. 2001: Pflanzensoziologische Exkursionsflora für Deutschland und angrenzende Gebiete, 8. Aufl. - Stuttgart.

Paulus, H. F. 2005: Zur Bestäubungsbiologie der Orchideen. - In: Blatt, H., Eccarius, W., \& Kretzschmar, H. (Hrsg.): Die Orchideen Deutschlands. - Uhlstädt-Kirchhasel.

RaAbe, U., Büscher, D., Fasel, P., Foerster, E., Götte, R., Haeupler, H, Jagel, A., Kaplan, K., Keil, P., Kulbrock, P., Loos, G. H., Neikes, N., Schumacher, W., Sumser, H., \& Vanberg, C. 2011: Rote Liste und Artenverzeichnis der Farn- und Blütenpflanzen - Pteridophyta et Spermatophyta - Nordrhein-Westfalen. - LANUV Nordrhein-Westfalen.

Scopoli, G. A. 1772: Flora Carniolica exhibens plantas Carnioliae indigenas et distributas in classes, genera, species, varietates, ordine Linnaeano. - Wien.

Siebert, H. 1998: Orchis tridentata - Aspekte der Ökologie einer nordhessischen Orchidee - Vortrag auf der 16. AHO-Vorstandstagung am 17. und 18.10.1998 in Eisenach.

Sundermann, H. 1980: Europäische und mediterrane Orchideen, 3. Aufl. - Hildesheim.

Tyteca, D. \& Klein, E. 2008: Genes, morphology and biology - the systematics of Orchidinae revisited. - J. Europ. Orchideen 40: 501-544.

Westrich, P. 2018: Die Wildbienen Deutschlands. - Stuttgart.

\section{Anschrift der Autoren}

Dr. Mathias Lohr, Technische Hochschule Ostwestfalen-Lippe, FB9, Landschaftsarchitektur und Umweltplanung, Campusallee 12, 32657 Lemgo,

E-Mail: mathias.lohr@hs-owl.de

Bernd Margenburg, Auf der Klause 5, 59192 Bergkamen, E-Mail: bernd@mgorch.de 